\title{
Avaliação da potência aeróbia de praticantes de Rugby em Cadeira de Rodas através de um teste de quadra
}

\author{
Lucinar Jupir Forner Flores ${ }^{1}$ \\ Luis Felipe Castelli Correia de Campos* 2 \\ Rafael Botelho Gouveia ${ }^{3}$ \\ Anselmo de Athayde Costa e Silva** 2 \\ Luís Gustavo de Souza Pena ${ }^{4}$ \\ José Irineu Gorla 2,3,4 \\ ${ }^{1}$ Curso de Educação Física Bacharelado e Laboratório de Avaliação do Desempenho no \\ Esporte, Universidade Estadual do Oeste do Paraná, Marechal Cândido Rondon, PR, Brasil \\ ${ }^{2}$ Grupo de Pesquisa em Avaliação Motora Adaptada, Faculdade de Educação Física da \\ UNICAMP, Campinas, SP, Brasil \\ ${ }^{3}$ Programa de Pós-Graduação da UNICAMP, Campinas, SP, Brasil \\ ${ }^{4}$ Departamento de Estudos da Atividade Física Adaptada, Faculdade de Educação Física da \\ UNICAMP, Campinas, SP, Brasil
}

\begin{abstract}
Resumo: O presente estudo teve por finalidade estimar a potência aeróbia em atletas com LME praticantes de RCR, correlacionar os níveis de $\mathrm{VO}_{2 \text { máx }}$ com a classificação funcional (CF) dos atletas e analisar 0 comportamento da FC antes e pós-teste. A amostra foi composta por 10 atletas com LME, do sexo masculino e idade média de 29,6 $\pm 6,5$ anos. Os atletas foram submetidos ao teste de corrida de 12 minutos e monitorados com o frequêncimetro cardíaco. A média do $\mathrm{VO}_{2 \operatorname{máx}}$ foi de $18,3 \pm 8,1 \mathrm{ml}(\mathrm{kg} \cdot \mathrm{min})^{-1}$ e da $\mathrm{FC}_{\text {máx }}$ de $114,6 \pm 25,3 \mathrm{bpm}$. Os valores de $\mathrm{VO}_{2 \text { máx }}$ obtidos no estudo são classificados como médio para a população com tetraplegia. Observou-se que existe correlação moderada $(r=0,77)$ entre a $\mathrm{CF}$ e o $\mathrm{VO}_{2 \text { máx }}$ entre atletas praticantes de RCR e por fim, observou-se baixos valores de FC frente á um teste submáximo, fato que pode estar relacionado à diminuição da atuação simpática após LME.
\end{abstract}

Palavras-chave: Esporte. Treinamento. Avaliação. Tetraplegia.

\section{Evaluation of the aerobic power of Wheelchair Rugby practitioners in a through a test court}

\begin{abstract}
The present study aimed to estimate aerobic power in wheelchair athletes with SCl. Other objetive was $\mathrm{VO}_{2 \max }$ levels correlate with the functional classification of athletes and analyze the behavior of the $\mathrm{FC}$ before and after testing. The sample consisted of 10 male athletes with $\mathrm{SCl}$ and mean age was $29.6 \pm 6.5$ years. The athletes were tested for race 12 minutes and the heart rate monitor was used. The average $\mathrm{VO}_{2 \max }$ was $18.3 \pm 8.1 \mathrm{ml}(\mathrm{kg} . \mathrm{min})^{-1}$, while the mean $\mathrm{HR}_{\max }$ was $114.6 \pm 25.3 \mathrm{bpm}$. The $\mathrm{VO}_{2 \max }$ values obtained in the study are classified as medium for people with quadriplegia. It was observed that there is a moderate correlation $(r=0.77)$ between the functional classification and $\mathrm{VO}_{2 \max }$ among athletes practicing WR and finally, was observed low values of HR will face a submaximal test, which may be related to decreased activity symphatetic after SCl.
\end{abstract}

Keywords: Sport. Training. Assessment. Quadriplegia.

\section{Introdução}

O Rugby em Cadeira de Rodas (RCR) é um esporte Paralímpico que foi criado no Canadá, no final da década de 70 . Inicialmente o esporte era uma alternativa para pessoas com limitações nos membros superiores e que levavam grande desvantagem na prática no Basquete em Cadeira de Rodas, devido ao alto grau de comprometimento (YILLA e SHERRIL, 1998). A prática do RCR pode ser realizada por atletas que

\footnotetext{
* Bolsista CNPq.

** Bolsista CAPES.
}

apresentam quadro de lesão da medula espinhal (LME), podendo ser total ou parcial acima da primeira vértebra torácica, ou seja, a tetraplegia, ou ainda pessoas com quadros de tetraequivalência (amputações de quatro membros, má-formação congênita e algumas síndromes que acometam o funcionamento motor dos 4 membros) (Comitê Paralímpico Brasileiro - $\underline{\mathrm{CPB}}$, 2011; INTERNATIONAL WHEELCHAIR RUGBY FEDERATION - IWRF, 2010). Para a participação no RCR é necessário uma classificação funcional para determinar o nível de comprometimento de cada atleta e também possibilitar a atuação de 
diferentes níveis de lesão e comprometimento, já que cada equipe é composta de 4 atletas em quadra e no somatório da classificação funcional dos 4 atletas não pode passar de 8 pontos em quadra. A classificação pontua as pessoas com menor comprometimento motor com 3.5 e os mais comprometidos com 0.5 , tendo classificações intermediárias de $(3 ; 2.5 ; 2 ; 1.5$ e 1 ponto) (IWRF, 2010).

O RCR se assemelha muito ao Rugby tradicional, por ter quase os mesmos objetivos e ser um jogo que envolve forte contato físico e necessidade de níveis elevados de condicionamento físico. $O$ jogo é desenvolvido em quadras de piso rígido, com as medidas do jogo de basquete de (15 metros de largura por 28 metros de comprimento). O RCR é considerado um esporte dinâmico com esforços intermitentes durante quatro tempos de 8 minutos cada, sendo fundamental que os atletas tenham agilidade para manusear a bola, acelerar, frear e direcionar a cadeira. (SARRO et al., 2010; GOOSEYTOLFREY; CASTLE; WEBBORN, 2006; YILLA e SHERRIL, 1998).

$\mathrm{Na}$ tetraplegia, devido à redução da atuação simpática e diminuição da massa muscular funcional, ocorre a redução da capacidade de trabalho, redução da frequência cardíaca máxima $\left(F C_{\text {máx }}\right)$ durante 0 exercício, e redução da capacidade cardiorrespiratória (capacidade e potência aeróbia), principais aspectos para 0 desenvolvimento da fadiga precoce (JACOBS; NASH, 2004; HAISMA et al., 2006). Nesse contexto, a potência aeróbia torna-se uma importante variável para participantes de jogos em cadeira de rodas (GOOSEY-TOLFREY; TOLFREY, 2008), visto que, o metabolismo aeróbio (fator predominante) nessas modalidades, será responsável em proporcionar uma recuperação metabólica mais rápida para os estímulos intensos (ㅂNTER et al., 2011). Sendo assim, a avaliação periódica da potência aeróbia em atletas praticantes de RCR é importante para análise das capacidades específicas do sistema respiratório, sanguíneo, circulatório e muscular, principalmente em atletas com deficiência física (LME) que possuem inúmeras alterações fisiológicas (TANHOFFER et al., 2012; LEICHT, BISHOP, GOSSEY-TOLFREY, 2011; BHAMBHANI, 2002).

$\mathrm{O}$ consumo máximo de oxigênio $\left(\mathrm{VO}_{2 \max }\right)$ é uma dos principais variáveis para a predição da potência aeróbia tanto em usuários de cadeira de rodas, quanto em indivíduos sem LME, sua importância é baseada no fato do nível de oxigênio no músculo durante a prática ser um fator limitante do desempenho. Ainda neste aspecto, vários autores indicam que os valores do

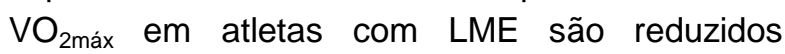
quando comparados as pessoas sem deficiência, devido à menor frequência cardíaca e ventilação (HOUNKER et al., 1998; LAKOMY; CAMPBELL; WILLIAMS, 1987; VINET et al., 2002), redução da circulação sanguínea para os músculos ativos no exercício, além de alterações do controle simpático (THIJSSEN; STEENDJIK; HOPMAN, 2009).

Avaliações físicas, com medidas e instrumentos laboratoriais em relação ao consumo máximo de $\mathrm{O}_{2}$ fornecem informações precisas e extremamente úteis para avaliação da capacidade aeróbia, entretanto, o alto custo dos equipamentos, as adaptações necessárias, o grande tempo para realização de testes individuais e procedimentos proibitivos para avaliações de grandes grupos tornam necessária à busca por avaliações em campos, pista e quadras para usuários em cadeira de rodas (GOSSEY-TOLFREY et al., 2008; VANLANDEWIJCK et al., 2006; FRANKLIN et al., 1990). Testes de campo e com fácil acessibilidade são atualmente muito importantes como ferramenta nas áreas de reabilitação e ciência do esporte no sentido de auxiliar na evolução do desempenho aeróbio em sedentários, atletas sendo usuários de cadeira de rodas atletas ou não (Vanderthommen et al., 2002; Vinet et al., 2002).

Apesar dos esforços para desenvolvimento de testes de campo/quadra, percebe-se timidamente estudos científicos com tabelas normativas (Janssen et al., 2002; Franklin et al., 1990) relacionados ao comportamento das variáveis fisiológicas para prescrição, controle e cuidados das sessões de treinamentos em participantes de esportes em cadeira de rodas. Um dos possíveis motivos é a falta da homogeneidade do padrão físico dos usuários de cadeira de rodas, devido à existência de diferentes níveis de lesões e comprometimentos, que implicam em diversas alterações neuromusculares, metabólicas e fisiológicas que precisam ser analisadas de forma específica para a prescrição de exercícios de forma organizada. 
Nesse sentido, o presente estudo teve por finalidade estimar a potência aeróbia em atletas com LME praticantes de RCR, correlacionar os níveis de $\mathrm{VO}_{2 \text { máx }}$ com a classificação funcional a partir dos resultados obtidos no teste submáximo de 12 minutos e analisar o comportamento da FC antes e pós-teste. Tais parâmetros são fundamentais no auxílio das prescrições de exercícios de forma individualizada.

\section{Materiais e Métodos}

A amostra do estudo foi composta por dez atletas praticantes de RCR, tetraplégicos com lesões completas e incompletas, sendo nove atletas com nível cervical (lesões medulares até C7) e apenas um com lesão torácica alta (nível T1). Todos os atletas do sexo masculino, com idade entre 21 e 41 anos, e mínimo de um ano de prática da modalidade. A descrição dos sujeitos segue na Tabela 1.

Tabela 1. Características dos sujeitos avaliados.

\begin{tabular}{ccccc}
\hline Sujeitos & Idade (anos) & Nível da Lesão & CF & TL \\
\hline A & 33,6 & C6-C7 & 1.5 & 6,2 \\
B & 22,8 & C7 & 2.5 & 6,3 \\
C & 24,6 & C6-C7 & 2.5 & 3,5 \\
D & 33,6 & C4-C5-C6 & 1.0 & 15,7 \\
E & 24,0 & C6 & 1.5 & 5,6 \\
F & 29,4 & T1\# & 3.5 & 7,2 \\
G & 36,2 & C5 & 0.5 & 13,7 \\
H & 21,0 & C6 & 2 & 5,4 \\
I & 41,1 & C5,C6 & 2 & 7,6 \\
J & 30,2 & C5,C6,C7 & 1.5 & 3,4 \\
\hline Média & $\mathbf{2 9 , 6}$ & - & & $\mathbf{7 , 5}$ \\
DP & $\mathbf{6 , 5}$ & - & & $\mathbf{4 , 1}$ \\
Mín. & $\mathbf{2 1}$ & - & & $\mathbf{3 , 4}$ \\
Máx. & $\mathbf{4 1 , 1}$ & - & $\mathbf{1 5 , 7}$ \\
\hline
\end{tabular}

LME: Lesão da Medula Espinhal, Mín: mínimo, Máx: máximo, CF: classificação funcional, TL: tempo de lesão, \#: O sujeito $\mathrm{F}$ apresenta lesão no nível neurológico torácico, mas devido ao comprometimento ocasionado pela mesma, após várias classificações funcionais para o jogo foi permitida sua participação no RCR (IWRF, 2010; ORR e MALONE, 2010).

O estudo foi submetido ao Comitê de Ética em Pesquisa da Faculdade de ciências médicas da UNICAMP e aprovado sob o no de protocolo no 276/2010. As coletas de dados foram realizadas no Ginásio da Faculdade de Educação Física da UNICAMP. Inicialmente, foram explicados os procedimentos a serem realizados e seus objetivos científicos aos participantes da pesquisa e posteriormente, foram realizadas a anamnese e a assinatura do termo de consentimento livre e esclarecido.

\section{Protocolo de Treinamento}

Os atletas foram submetidos ao treinamento dentro da proposta de Matveev (1981), de carga distribuída, com duas competições alvo durante o ano. O período utilizado para a avaliação dos atletas foi após a fase de transição, em virtude do inicio do segundo período de preparação para a disputa do segundo campeonato pré-estabelecido pelo planejamento (período preparatório).

As sessões de treinamento consistiam em treinamentos físicos associados aos aspectos técnicos e/ou táticos, com duração média de uma hora e meia por sessão e com frequência semanal de três vezes.

\section{Procedimentos}

Para a coleta de dados em relação à massa corporal e estatura, foram utilizados uma balança de marca Filizola ${ }^{\circledR}$ com precisão de $0,1 \mathrm{~kg}$, um estadiômetro horizontal com precisão de $0,1 \mathrm{~cm}$ e dois colchonetes para apoio dos sujeitos ao solo. Para a coleta da massa corporal e estatura foram utilizados os procedimentos descritos por Gorla et al. (2009), nos quais os sujeitos são posicionados sentados e deitados, respectivamente.

Em seguida, as cintas do frequêncimetro cardíaco da marca Polar $^{\circledR}$ (Finlândia) modelo RS800CX foram instaladas na região torácica dos atletas. As cintas via transmissão sem fio codificavam as informações e transmitiam ao relógio para registro e monitoramento dos valores da frequência cardíaca antes, durante e após a realização do teste. Como adaptação para melhor fixação da cinta do equipamento foram colocadas tiras de fita esparadrapo na região anterior e na 
posição horizontal entre a cinta e a derme dos atletas.

Para o teste de estimativa da potência aeróbia, foram utilizadas cadeira de rodas esportivas específicas da modalidade, quadra de piso rígido não-escorregadio, oito cones e dois cronômetros. O teste para estimativa da potência aeróbia foi realizado conforme protocolo proposto por Franklin et al. (1990), no qual, é delimitado um retângulo, na quadra de piso rígido não escorregadio, com medidas de 25 × 15 metros. Neste retângulo, foram colocados cones em cada uma das extremidades e também a cada 2 metros de seus respectivos vértices, de maneira a se obter um perímetro de 75,32 metros. Para a realização do teste, os sujeitos deveriam percorrer a maior distância possível, em torno do retângulo delimitado na quadra (Figura 1), sentados nas cadeiras de rodas esportivas, no período de 12 minutos.

Após a realização do teste de quadra foi calculada a distância em metros percorrida por cada atleta em sua cadeira de roda esportiva e em seguida foi transformado os valores em metros para milhas para a inserção dos valores de todos os usuários na equação proposta por Franklin et al. (1990) a seguir: $\mathrm{VO}_{2 \operatorname{máx}}(\mathrm{ml} / \mathrm{kg} / \mathrm{min}$ ) $=$ (Distância milhas - 0,37)/0,0337, cujo coeficiente de confiabilidade é de $r=0,84$ $(p<0,001)$.

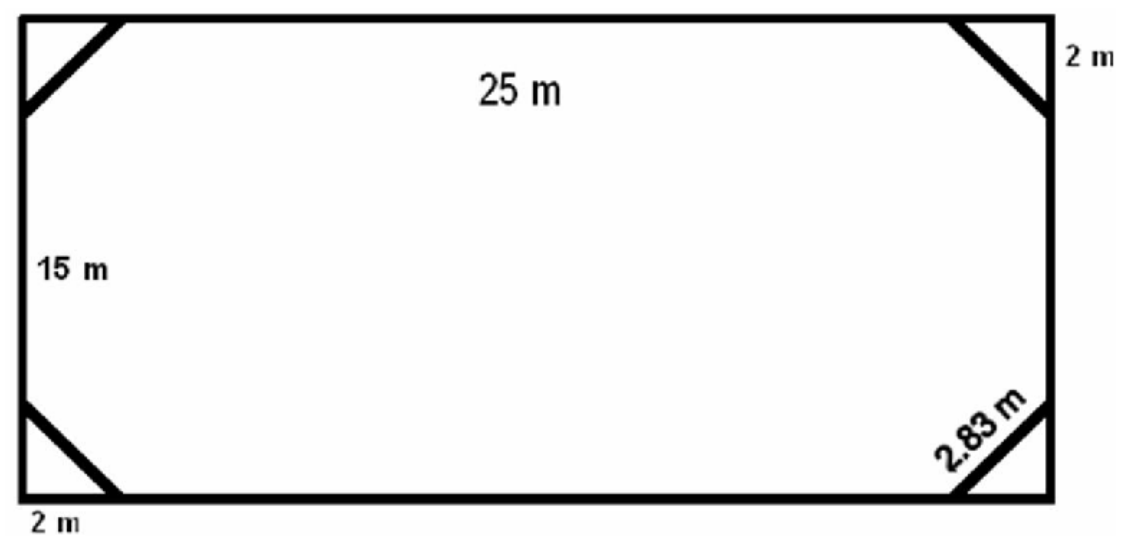

Figura 1. Circuito delimitado em quadra para a realização do teste de potência aeróbia

A partir dos resultados obtidos os atletas foram classificados de acordo com os parâmetros normativos propostos por Franklin et al. (1990). Os referidos valores são descritos no Quadro 1.

Quadro 1. Classificação da potência aeróbia (estimada) proposta por Franklin et al. (1990).

\begin{tabular}{|ccc|}
\hline Distância (milhas/metros) & $\begin{array}{c}\mathbf{V O}_{\mathbf{2 m a ́ x}} \\
\mathbf{m l} / \mathbf{k g} / \mathbf{m i n}\end{array}$ & Nível de condicionamento \\
\hline$<0,63 /<1,013$ & $<7,7$ & Pobre \\
$0,6-0,86 / 1,0129-1,384$ & $7,7-14,5$ & Abaixo da média \\
$0,87-1,35 / 1,400-2,17$ & $14,6-29,1$ & Médio \\
$1,36-1,59 / 2,188-2,558$ & $29,2-36,2$ & Bom \\
$\geq 1,60 / \geq 2,574$ & $\geq 36,3$ & Excelente \\
\hline
\end{tabular}

\section{Análise Estatística}

Os dados foram analisados através de estatística descritiva (média, desvio padrão, mínimo e máximo). Em seguida, verificou-se a normalidade dos dados de frequência cardíaca através dos coeficientes de assimetria e curtose conforme proposição de Hair et al. (2009), pois segundo eles, os testes estatísticos Shapiro-Wilk e Kolmogorov-Smirnov, que avaliam a normalidade, são muito sensíveis para amostras menores de 30 sujeitos. Utilizou-se a ANOVA one way com post hoc Student-Newman-Keuls para a comparação dos dados de frequência cardíaca ao longo da realização do teste de 12 minutos/recuperação e a correlação de Pearson para relacionar os valores de $\mathrm{VO}_{2 \text { máx }}$ com a classificação funcional dos sujeitos. Valores de $p$ $<0,05$ foram considerados significativos para análises de comparação.

\section{Resultados}

Conforme objetivo do estudo de estimar a potência aeróbia de praticantes de Rugby em Cadeira de Rodas através de um teste de 12 
minutos em quadra, os resultados antropométricos são apresentados na Tabela 2.

Os resultados da distância percorrida em metros durante o teste de 12 minutos para avaliação da estimativa da potência aeróbia são apresentados na Tabela 3, assim como os valores de FC durante o repouso (FC rep), ao final do teste ( $F C$ final), 3 minutos após o final do teste (FC $3{ }^{`}$ ) e 5 minutos após o final do teste (FC 5`).

Tabela 2. Dados antropométricos das pessoas com lesão da medula espinhal.

\begin{tabular}{|c|c|c|c|}
\hline Sujeitos & Massa (kg) & Estatura (m) & IMC $\left(\mathrm{kg} / \mathrm{m}^{2}\right)$ \\
\hline$A$ & 60,6 & 1,76 & 19,57 \\
\hline B & 66,74 & 1,72 & 22,56 \\
\hline C & 56,26 & 1,57 & 22,83 \\
\hline D & 72,64 & 1,85 & 21,23 \\
\hline $\mathrm{E}$ & 61,71 & 1,75 & 20,15 \\
\hline$F$ & 57,23 & 1,69 & 20,04 \\
\hline G & 62,59 & 1,70 & 21,66 \\
\hline $\mathrm{H}$ & 75,16 & 1,83 & 22,44 \\
\hline I & 67,78 & 1,79 & 21,15 \\
\hline $\mathrm{J}$ & 64,56 & 1,86 & 18,66 \\
\hline Média & 64,5 & 1,75 & 21 \\
\hline DP & 6,2 & 0,09 & 1,4 \\
\hline Mín. & 56,2 & 1,57 & 18,66 \\
\hline Máx. & 75,1 & 1,86 & 22,83 \\
\hline
\end{tabular}

IMC: índice de massa corporal, DP: desvio-padrão, Mín: mínimo, Máx: máximo.

Tabela 3. Resultados e classificação dos sujeitos no teste de estimativa da potência aeróbia.

\begin{tabular}{ccccccccc}
\hline Sujeitos & $\begin{array}{c}\text { Distância } \\
(\mathbf{m t s})\end{array}$ & $\begin{array}{c}\text { Distância } \\
\text { (milhas) }\end{array}$ & $\begin{array}{c}\text { VO }_{\mathbf{2 m a ́ x}} \\
(\mathbf{m l / k g} / \mathbf{m i n})\end{array}$ & $\begin{array}{c}\text { Classificação } \\
\text { (Franklin et al. } \\
\mathbf{1 9 9 0})\end{array}$ & $\begin{array}{c}\text { FC } \\
\text { rep. }\end{array}$ & FC final $^{\text {FC 3' }}$ & FC 5' $^{\prime}$ \\
\hline A & 1737,36 & 1,08 & 21,20 & Médio & 85 & 130 & 100 & 93 \\
B & 2066,8 & 1,29 & 27,30 & Médio & 72 & 123 & 100 & 104 \\
C & 2187,78 & 1,37 & 29,54 & Bom & 74 & 176 & 112 & 103 \\
D & 821,2 & 0,51 & 4,23 & Pobre & 81 & 102 & 93 & 87 \\
E & 1525,14 & 0,95 & 17,27 & Médio & 72 & 88 & 66 & 64 \\
F & 2033,64 & 1,27 & 26,69 & Médio & 70 & 110 & 155 & 144 \\
G & 1209,02 & 0,75 & 11,42 & Abaixo da média & 61 & 93 & 69 & 65 \\
H & 1646,72 & 1,03 & 19,52 & Médio & 104 & 113 & 100 & 100 \\
I & 1251,78 & 0,78 & 12,21 & Abaixo da mdia & 73 & 115 & 110 & 100 \\
J & 1315,44 & 0,82 & 13,39 & Abaixo da média & 75 & 96 & 91 & 82 \\
\hline Média & 1579,5 & 0,99 & 18,3 & Médio & 76.7 & $114.6^{*}$ & $99,6^{*}$ & $94,2^{*} \#$ \\
DP & 439,1 & 0,3 & 8,1 & - & 11.5 & 25.3 & 24,8 & 22,8 \\
\hline
\end{tabular}

FC: frequência cardíaca em bpm, FC3: frequência cardíaca após três minutos de recuperação, FC5: frequência cardíaca após cinco minutos de recuperação. * $p<0,05$ vs repouso, \# $p<0,05$ vs final.

Após a obtenção dos resultados em metros do teste, transformou-se estes resultados em milhas para o cálculo dos valores de estimativa do $\mathrm{VO}_{2 \text { máx }}(\mathrm{ml} / \mathrm{kg} / \mathrm{min})$ conforme protocolo proposto por Franklin et al., (1990). A classificação individualizada da potência aeróbia estimada encontra-se na Tabela 3.

Os resultados de FC ao final do teste de 12 min e ao longo da recuperação foram maiores e significativamente diferentes quando comparados com os valores de repouso. $E$ com 5 minutos de recuperação os valores de FC foram diferentes dos resultados ao final do teste de $12 \mathrm{~min}$.
Também, testou-se a relação entre a potência aeróbia e a classificação funcional proposta pela RCR, para verificar se na amostra estudada existe 0 indício de que quanto maior o comprometimento (lesão mais alta geralmente) menor a capacidade de realizar exercícios, aqui evidenciado pelo índice de consumo máximo de oxigênio. A correlação de Pearson foi utilizada para testar a relação entre as variáveis e ficou comprovada a relação entre consumo máximo de oxigênio, no qual, quanto maior o consumo de oxigênio maior a classificação funcional e menor o comprometimento do lesado medular respectivamente $(r=0,77 ; p<0,05)$. A Figura 02 ilustra este resultado: 


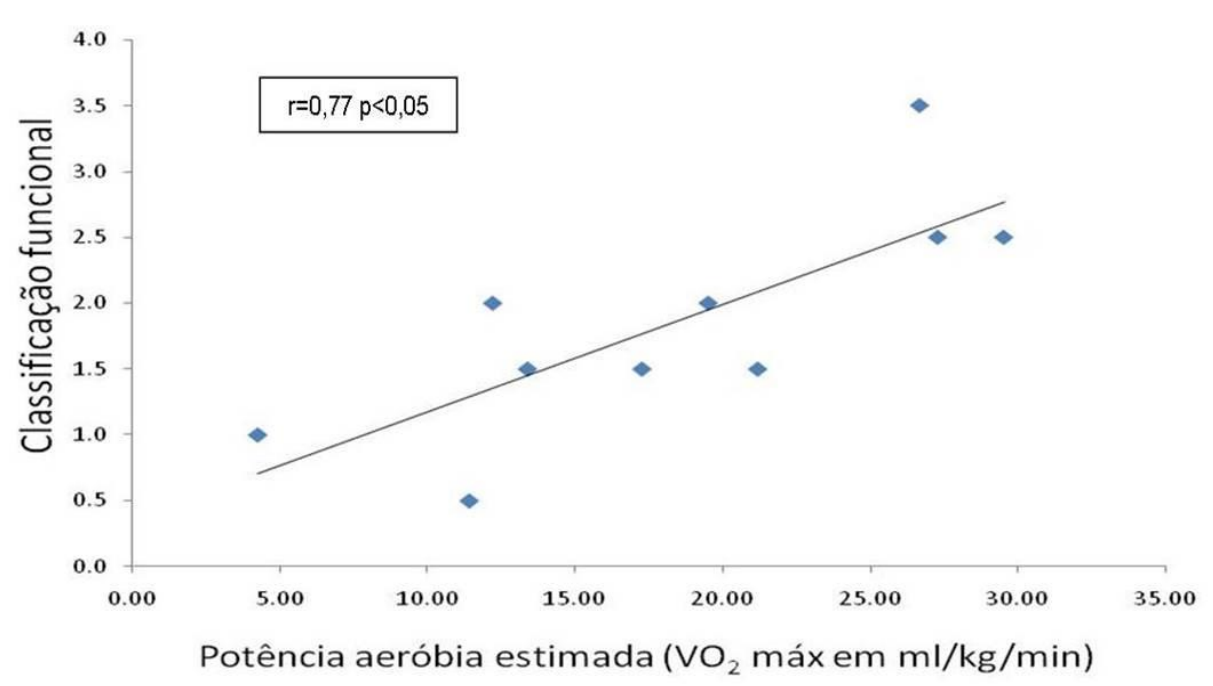

Figura 2. Correlação entre a classificação funcional e os valores de potência aeróbia

\section{Discussão}

Com o objetivo de estimar a potência aeróbia de pessoas com lesão da medula espinhal praticantes de RCR foi utilizado um teste para usuários de cadeira de rodas. A escolha por este protocolo foi devido ao fato de possuir boa correlação $(r=0,84 ; p<0,001)$ com a medida direta de $\mathrm{VO}_{2 \text { máx }}($ Franklin et al., 1990). Além disso, o protocolo possibilita que sujeitos tetraplégicos consigam realizar o protocolo, pois a grande maioria dos protocolos com ergômetros de braços não são adaptáveis para tal população, pois PLME tetraplégicas possuem muito comprometimento do tronco e com isto não se mantém estáveis sobre a maioria dos ciclo ergômetros,

Este protocolo tem ainda o aspecto favorável de não necessitar de sinal sonoro específico durante a execução do teste, como nos testes conhecidos como Beeptest, os quais, no Brasil ainda não foram validades. Outro ponto relevante é que nos testes Beep o sujeito avaliado tem que executar uma volta de $180^{\circ}$ e no protocolo utilizado neste estudo a volta é de $90^{\circ}$. Isto facilita a execução pelos sujeitos tetraplégicos que possuem comprometimento postural. Acredita-se que as curvas de $180^{\circ}$ podem dificultar a realização do teste fazendo com que o resultado expresse o grau de comprometimento da força/potência muscular localizada em detrimento da condição aeróbia dos avaliados, que é o principal objetivo do teste.

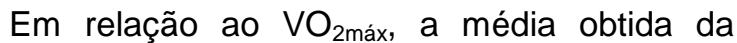
amostra do estudo foi de $18,3 \pm 8,1 \mathrm{ml}$ (kg.min) ${ }^{-1}$ classificado conforme tabela de Franklin et al. (1990) como valores de condicionamento físico "médios" para usuários de cadeira de rodas em geral, sendo cinco sujeitos com nível de condicionamento "médio", três "abaixo da média" e um com classificação "pobre" e um nível "ótimo". Corroborando os valores obtidos no estudo em relação ao $\mathrm{VO}_{2 m a ́ x}$, observa-se que os resultados foram superiores ao valores encontrados por Goosey-Tolfrey; Castle; Webborn, (2006) no qual os participantes eram de 2 modalidades esportivas (tênis em cadeira de rodas e RCR), próximos ao estudo de Barfield et al. (2010) e valores inferiores aos testes de ergômetro de cadeira de rodas realizados por Janssen et al. (2002) e no teste de esteira em cadeira de rodas realizados por Morgulec et al. (2006).

No estudo de Morgulec et al. (2006) que avaliaram 14 jogadores da Liga Polonesa de RCR em dois anos consecutivos (2003 e 2004) para verificar o efeito do treinamento no desempenho aeróbio realizado ao longo deste período, as avaliações foram realizadas em esteira adaptada para usuários de cadeira de rodas e avaliação do componente aeróbio foi feita através de análise direta de gases. O valor médio do $\mathrm{VO}_{2 \text { máx }}$ encontrado em 2003 foi de $19,9 \pm 4,9 \mathrm{ml} / \mathrm{kg} / \mathrm{min}$ e no ano seguinte, após um ano de treinamento os valores foram significativamente maiores $(27,4 \pm 4,8 \mathrm{ml} / \mathrm{kg} / \mathrm{min})$. Este incremento foi justificado pelos autores, pelas possíveis 
alterações da melhora da capacidade de resistência funcional, bem como melhora nos fatores de risco como: redução de riscos de hipertensão, obesidade e acidente vascular encefálico. Indivíduos tetraplégicos praticantes de esportes têm tendência à redução da estimulação simpática reduzindo os efeitos circulatórios dos exercícios (WELLS; HOOKER, 1990). No entanto Eggers et al. (2001) indicam que não está totalmente claro se estes efeitos do treinamento foram devidos a alterações centrais (volume de ejeção, debito cardíaco e ou ventilaçào minuto) ou fatores periféricos (atividade enzimáticas, diferença artério-venosa).

Tanto no estudo de Morgulec et al. (2006) quanto no de Janssen et al. (2002), os valores médios de $\mathrm{VO}_{2 \text { máx }}$ encontrados são superiores aos encontrados neste estudo, isto pode ser explicado pela forma de avaliação, já que os estudos aqui citados avaliaram o $\mathrm{VO}_{2 \text { máx }}$ de forma direta enquanto que, o que apresentamos neste estudo é o $\mathrm{VO}_{2 \operatorname{máx}}$ estimado. Além disso, Janssen et al. (2002) utilizaram em sua amostra, atletas com paraplegia, que além de apresentarem menor comprometimento funcional e aeróbio, podem ter através do treinamento, os níveis de potência e capacidade aeróbia aumentada significativamente em relação aos tetraplégicos.

JACOBS; NASH; RUSINOWSKI, (2001) avaliaram os efeitos do treinamento em circuito (12 semanas) sobre a capacidade cardiorrespiratória e força muscular em paraplégicos. Aumentos significativos foram observados no consumo máximo de oxigênio $(29,7 \%, p<0,01)$, e na potência de pico durante o teste em ergômetro de braço $(p<0,05)$.

Outro estudo importante aplicando o treinamento físico como situação de intervenção em pessoas com lesão da medula espinhal foi o de Bougenot et al. (2003). Neste estudo com sete indivíduos paraplégicos não treinados do sexo masculino foram avaliados após um programa de treinamento com duração de 6 semanas em esteira própria para cadeira de rodas, e análises sobre variáveis cardiorrespiratórias em teste ergométrico foram estudadas. Os resultados obtidos mostraram aumentos significativos das variáveis $\mathrm{VO}_{2 \text { máx }}$ e potência pico (watts).

Além do parâmetro de $\mathrm{VO}_{2 \text { máx }}$ supracitado, Barfield et al. (2010) investigaram a intensidade de treinamento durante o RCR. Eles avaliaram 9 jogadores de RCR americanos através de analisador de gases em ergômetro de braço e com frequêncimetro para determinar os valores de FC e FC de reserva como parâmetro de intensidade dos treinamentos do RCR. A FC máx chegou a valor médio de $118 \pm 17$ batimentos no grupo estudado. Interessante neste estudo foi a análise da FC durante as 3 sessões de treinamento, no qual foram divididas as principais ações dos jogadores de RCR (embalar a cadeira continuamente, embalar com trocas de direção/contatos de jogo, passes e giros e situação de coletivo/jogo) e os valores médios de FC encontrados nestas situações foi de $105 \pm 13$ bpm.

Através desse estudo os autores indicam que os praticantes de RCR apresentaram platô em relação à FC durante o exercício, resultado este que pode ser deduzido pela limitação do débito cardíaco, transporte e consumo de $\mathrm{O}_{2}$ e da capacidade funcional da prática dos exercícios nesta população (BIRK et al., 2001; BRAVO et al., 2004).

Limitações periféricas também podem confundir respostas agudas, além da disponibilidade limitada da massa muscular e a redução da atividade da bomba muscular serem grandes inibidores da capacidade máxima de exercício (BIRK et al., 2001). Os resultados deste estudo indicam que os participantes de RCR podem se exercitar em uma intensidade $(70 \%$ da FC de reserva) para que provoque melhorias na aptidão cardiorrespiratória, dada concepção adequada das condições de treinamento, sem manipulação médica ou terapêutica.

Os resultados de FC máxima em nosso estudo foram semelhantes aos citados nesta discussão. Normalmente indivíduos tetraplégicos apresentam bradicardia de repouso e pouca resposta de FC ao aumento da intensidade do exercício físico, isto acontece devido a LME, principalmente quando a lesão é completa e em menor grau na lesão incompleta da medula espinha, (interromper ou diminuir a atividade simpática cardíaca). $\mathrm{Na}$ amostra deste estudo houveramtivemos participantes com lesão incompleta e seus valores de $\mathrm{FC}_{\text {final }}$ foram semelhantes ao de indivíuos sem deficiência física, supondo que provávelmente a lesão medular não prejudicou esta importante função, o que propicia melhores resultados relacionados a função cardíaca em exercício e as variáveis de capacidade aeróbias, não por acaso o indivíduo com maior valor de potência aeróbia 
estimada, foi um sujeito com lesão incompleta da medula espinhal.

Vários autores associam a prática regular de exercício físico com múltiplos resultados positivos entre indivíduos com LME, incluindo a melhora da capacidade cardiorrespiratória (JACOBS; NASH, 2004; JACOBS; NASH; RUSINOWSKI, 2001), diminuição da aterosclerose sub-clínica (MATOSSOUZA et al., 2012), melhora da capacidade muscular (JANSSEN et al., 2002), melhora funcional (STREADWARD, 1998), redução do esforço ao realizar atividades da vida diária (JANSSEN et al., 1996), além de redução do risco de doenças secundárias (KOSMA; CARDINAL; RINTALA, 2002).

\section{Considerações Finais}

A potência aeróbia estimada de pessoas com lesão da medula espinhal (tetraplégicos) praticantes de Rugby em cadeira de rodas mostrou-se dentro da normalidade para esta população. O protocolo usado propicia um quadro de referência e neste quadro o valor médio do grupo indicou o nível bom - algo mediano na escala desenvolvida por Franklin et al., (1990). Foi observada correlação moderada quando comparados os valores de classificação funcional com os níveis de $\mathrm{VO}_{2 m a ́ x}$, sugerindo que, quanto maior o nível de classificação funcional, menor o comprometimento motor e maiores valores de aptidão cardiorrespiratória. Por fim, estudos que apresentaram valores superiores aos encontrados em nosso estudo foram realizados com avaliação ergoespirométrica direta. Isto pode ser um fator importante para novas avaliações.

\section{Referências}

BARFIELD, J. P.; MALONEB, L. A.; ARBOC, C.; JUNG, A. P. Exercise intensity during wheelchair rugby training. Journal os Sports Science. Londres, v. 28, n. 4, p. 389-398, 2010. DOI: http://dx.doi.org/10.1080/02640410903508839>.

BHAMBHANI, Y. Physiology of wheelchair racing in athletes with spinal cord injury. Sports

Medicine, Auckland, v.32, n.1, p. 23-51, 2002.

BIRK, T. J.; NIESHOFF, E.; GRAY, G.; STEEBY, J.; JABLONSKI, K. Metabolic and cardiopulmonary responses to acute progressive resistive exercise in a person with $\mathrm{C} 4$ spinal cord injury. Spinal Cord, Edegem, v.39, p. 336-339, 2001. DOI: <http://dx.doi.org/10.1038/sj.sc.3101164>.
BOUGENOT, M.P.; TORDI, N.; BETIK, A.C.; MARTIN, X.; LE FOLL, D.; PARRATTE, B.; LONSDORFER, J.; ROUILLON, J.D. Effects of a wheelchair ergometer training programme on spinal cord-injured persons. Spinal Cord, Edegem, v. 41, p. 451-456, 2003. DOI: <http://dx.doi.org/10.1038/sj.sc.3101475>.

BRAVO, G.; GUIZAR-SAHAGUN, G.; IBARRA, A.; CENTURION, D.; VILLALON, C. M.

Cardiovascular alterations after spinal cord injury:

An overview. Current Medicinal ChemistryCardiovascular \& Hematological Agents, Oak Park, v.2, n.2, p.133-148, 2004. DOI: <http://dx.doi.org/10.2174/1568016043477242>.

COMITÊ PARAOLÍMPICO BRASILEIRO - CPB. Rúgbi em cadeira de rodas. Disponível em: <http://www.cpb.org.br/esportes/modalidades/rugb i-em-cadeira-de-rodas >. Acesso em: 22/04/2011.

EGGERS, L.; CARSON, C.; EVANS, K.; SWANK, A.M.; ADAMS, K.J.; BARNARD, K. L.; BERNING, J.M. Limits factors for increasing $\mathrm{VO}_{2}$ peak for individuals with spinal cord injury. Clinical Exercise Physiology, Indianapolis, v.3, n.1, p. 10-16, 2001.

FRANKLIN, B.A.; SWANTEK, K.I.; GRAIS, S.L.; JOHNSTONE, K.S.; GORDON, S.; TIMMIS, G.C. Field test estimation of maximal oxygen consumption in wheelchair users. Archives of Physical Medicine and Rehabilitation, Reston, v.71, n. 8, p. 574-578, 1990.

GOOSEY-TOLFREY, V., CASTLE, P.,

WEBBORN, N. Aerobic capacity and peak power output of elite quadriplegic games players. British Journal of Sports Medicine, Londres, v. 40, n.8, p. 684-687, 2006. DOI:

<http://dx.doi.org/10.1136/bjsm.2006.026815>.

GOOSEY-TOLFREY, V., TOLFREY, K. The multistage fitness test as a predictor of endurance fitness in wheelchair athletes. Journal of sports sciences, Londres, v.26, n. 5, p. 511-517, 2008. DOI:

http://dx.doi.org/1010.1080/02640410701624531.

GORLA, J. I. CAMPANA, M. B., OLIVEIRA, L. Z. Teste e avaliação em esporte adaptado. São Paulo: Phorte, 2009.

HAIR , J.F.; BLACK, W. C.; BABIN, B. J.; ANDERSON, R. E.; TATHAN, R. L. Análise Multivariada de dados. 6ㄹ $\mathrm{Ed}$. Porto Alegre: Bookmann, 2009.

HAISMA, J.A.; VAN DER WOUDE, L.H.V.; STAM, H.J.; BERGEN, M.P.; SLUIS, T.A.R.;

BUSSMANN, J.B.J. Physical capacity in wheelchair-dependent persons with a spinal cord injury: a critical review of the literature. Spinal 
Cord, Edegem, v. 44, p. 642-652, 2006. DOI: $<$ http://dx.doi.org/10.1038/sj.sc.3101915>.

HOUNKER, M.; SCHMID, A.; SORICHTER, S.; SCHMIDT TRUCKSÄB, A.; MROSEK ,P.; KEUL, J. Cardiovascular differences between sedentary and wheelchair trained subjects with paraplegia. Medicine Science and Sports and Exercise, Indianapolis, v. 30, p. 609-613, 1998.

HUNTER, G. R., HUTZLER, Y. S., MECKEL, Y., BERZEN, J. Aerobic and anaerobic power. In: VANLANDEWIICK, Y. C., THOMPSON, W. R. The Paralympic Athletes. Handbook of Sports Medicine and Science. Wiley-Blackwell. 2011.

INTERNATIONAL WHEELCHAIR RUGBY FEDERATION. IWRF Classification Manual. Disponível em:

$<$ http://www.iwrf.com/classification.html >. Acesso em: 10/12/2010.

\section{JACOBS, P.; NASH, M. Exercise}

recommendations for individuals with spinal cord injury. Sports Medicine, Auckland, v.34, p. 725751, 2004.

JACOBS, P.L.; NASH, M.S.; RUSINOWSKI, J.W. Circuit training provides cardiorespiratory and strength benefits in persons with paraplegia. Medicine Science and Sports and Exercise, Indianapolis, v.33, p. 711-717, 2001.

JANSSEN, T.W.J.; DALLMEIJER, A.J.; VEEGER, D.H.E.J.; VAN DER WOUDE, L.H.V. Normative values and determinants of physical capacity in individuals with spinal cord injury. Journal of Rehabilitation Research and Development, Baltimore, v.39, n.1, p. 29-39, 2002.

JANSSEN, T.W.J.; VAN OERS, C.A.J.M.; ROZENDAAL, E.P.; WILLEMSEN, E. M.; HOLLANDER, A.P.; VAN DER WOUDE, L.H.V. Changes in physical strain and physical capacity in men with spinal cord injuries. Medicine Science and Sports and Exercise, Indianapolis, v. 28, n.5, p. 551-559, 1996.

KOSMA, M.; CARDINAL, B. J.; RINTALA, P. Motivating individuals with disabilities to be physically active. Quest, Londres, v.54, n.2, 116132, 2002.

LAKOMY, H.K.A.; CAMPBELL, M.A.I.; WILLIAMS, C. Treadmill performance and selected physiological characteristics of wheelchair athletes. British Journal of Sports Medicine, Londres, v. 21, n. 3, p. 130-133, 1987. DOI: $<$ http://dx.doi.org/10.1136/bjsm.21.3.130>.

LEICHT, C. A., BISHOP, N. C., GOOSEYTOLFREY, V. L. Submaximal exercise responses in tetraplegic, paraplegic and non spinal cord injured elite wheelchair athletes. Scandinavian journal of Medicine \& Science in Sports, Chichester, v.22, n.4, p.1-8, 2011.

MATOS-SOUZA, J.; COSTA E SILVA, A. A.; CAMPOS, L. F. C. C; GOULART, D.; SCHEREIBER, R.; ROSSI, G.; PIOMAGALHÃES, J.; ETCHEBEHERE, M.; GORLA, J.I.; CLIQUET-JR, A.; NADRUZ JR, W. Physical activity is associated with improved subclinical atherosclerosis in spinal cord injury subjects independent of variation in traditional risk factors. International Journal of Cardiology, Norwich, 2012. DOI:

<http://dx.doi.org/10.1016/j.ijcard.2012.09.222>.

MATVEEV LP. Fundamental of sport training. Moscow: Progress Publishers, 1981.

MORGULEC, N.; KOZMOL, A.; MOLIK, B.; HÜBNER-WOŹNIAK, E.; RUTKOWSKA, I. The effect of training on aerobic performance in wheelchair rugby players. Agencja Wydawnicza Medsportpress, Varsóvia, v. 12, n. 2, pp. 195198, 2006.

ORR, K.; MALONE, L.A. Wheelchair Rugby. In GOOSEY-TOLFREY, V. (org.). Wheelchair Sport: a complete guide for athletes, coaches and teachers. Champaign: Human Kinetics, 2010.

SARRO, K.J.; MISUTA, M.S.; BURKETT, B.; MALONE, L.A.; BARROS, R.M.L. Tracking of wheelchair rugby players in 2008 Demolition Derby final. Journal of Sports Sciences, Londres, v.28, n.2, p.193-200, 2010.

TANHOFFER, R. A., TANHOFFER, A. I. P., RAYMOND, J., HILLS, A. P., DAVIS, G. M. Comparison of methods to assess energy expenditure and physical activity in people with spinal cord injury. Journal of Spinal Cord Medicine, Cleveland, v.35, n.1, p.35-45, 2012. DOI:

http://dx.doi.org/10.1179/2045772311Y.00000000 $\underline{46}>$.

THIJSSEN, D. H. J.; STEENDIJK, S; HOPMAN, M. T. E. Blood redistribution during exercise in subjects with spinal cord injury and controls. Medicine Science and Sport and Exercise, Indianapolis, v. 41, n.6, p. 1249-1254, 2009. DOI: $<$ http://dx.doi.org/10.1249/MSS.0b013e318196c90 2>.

VANDERTHOMMEN, M., FRANCAUX, M., COLINET, C., LEHANCE, C., LHERMEROUT, C., CRIELAARD, J. M., THEISEN, D. A multistage field test of wheelchair users for evaluation of fitness and prediction of peak oxygen consumption. Journal of Rehabilitation Research and Development, Baltimore, v.39, n.6, p.685-692, 2002. 
VANLANDEWIJCK, Y., VLIET, P. V., VERELLEN, J., THEISEN, D. Determinants of shuttle run performance in the prediction of peak $\mathrm{VO}_{2}$ in wheelchair users. Disability and Rehabilitation, Suffolk, v.28, n.20, p.1259-1266, 2006.

VINET, A., GALLAIS, D. L., BOUGES, S., BERNARD, P.L., POULAIN, M., VARRAY, A., MICALLEF. Prediction of $\mathrm{VO}_{2 \text { peak }}$ in wheelchairdependent athlets from the adapted Leger and Boucher test. Spinal Cord, Edegem, v.40, n.10, p.507-512, 2002. DOI: <http://dx.doi.org/10.1038/sj.sc.3101361>.

WELLS, C.L.; HOOKER, S.P. The Spinal Injured Athlete. Adapted Physical Activity Quartely, Champaign, v.7, n. 3, p. 265-285, 1990.

YILLA, A. B.; SHERRIL, C. Validating the Beck battery of quad rugby skills tests. Adapted Physical Activity Quartely, Champaign, v.15, n.2, p.155-167, 1998.

Apoio: Através de bolsas de mestrado e doutorado CNPq e CAPES.

Comitê de Ética: Comitê de Ética em Pesquisa da Faculdade de ciências médicas da UNICAMP

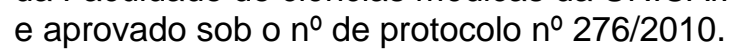

\section{Endereço:}

Lucinar Jupir Forner Flores

Av. Arnaldo Busatto, 210 apto 102, Centro

Santa Helena PR Brasil

85892-000

Telefone: (45) 3284-7855/ (45) 9919-5226

e-mail: lucinarflores@yahoo.com.br

Recebido em: 28 de novembro de 2011.

Aceito em: 20 de abril de 2013. 